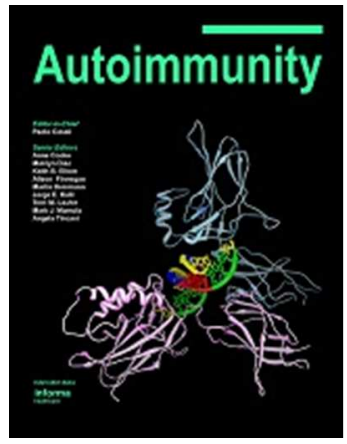

\title{
Autoimmune, rheumatic, chronic inflammatory diseases: Neutrophil Extracellular Traps on Parade
}

\begin{tabular}{|c|c|}
\hline Journal: & Autoimmunity \\
\hline Manuscript ID & GAUT-2018-0074.R1 \\
\hline Manuscript Type: & Review Article \\
\hline Date Submitted by the Author: & $n / a$ \\
\hline Complete List of Authors: & $\begin{array}{l}\text { Podolska, Malgorzata; Friedrich-Alexander University of Erlangen- } \\
\text { Nuremberg, Erlangen University Hospital, Institute for Clinical Immunology } \\
\text { and Rheumatology, Department for Internal Medicine } 3 \\
\text { Mahajan, Aparna; Friedrich-Alexander University of Erlangen-Nuremberg, } \\
\text { Erlangen University Hospital, Institute for Clinical Immunology and } \\
\text { Rheumatology, Department for Internal Medicine } 3 \\
\text { Knopf, Jasmin; Friedrich-Alexander University of Erlangen-Nuremberg, } \\
\text { Erlangen University Hospital, Institute for Clinical Immunology and } \\
\text { Rheumatology, Department for Internal Medicine } 3 \\
\text { Hahn, Jonas; Friedrich-Alexander University of Erlangen-Nuremberg, } \\
\text { Erlangen University Hospital, Institute for Clinical Immunology and } \\
\text { Rheumatology, Department for Internal Medicine } 3 \\
\text { Boeltz, Sebastian; Friedrich-Alexander University of Erlangen-Nuremberg, } \\
\text { Erlangen University Hospital, Institute for Clinical Immunology and } \\
\text { Rheumatology, Department for Internal Medicine } 3 \\
\text { Munoz, Luis; Friedrich-Alexander University of Erlangen-Nuremberg, } \\
\text { Erlangen University Hospital, Institute for Clinical Immunology and } \\
\text { Rheumatology, Department for Internal Medicine } 3 \\
\text { Bilyy, Rostyslav; Danylo Halytsky Lviv National Medical University } \\
\text { Herrmann, Martin; Friedrich-Alexander University, Internal Medicine } 3\end{array}$ \\
\hline Keywords: & $\begin{array}{l}\text { Neutrophil Extracellular Traps, Rheumatic diseases, systemic lupus } \\
\text { erythematosus, rheumatoid arthritis, anti-phospholipid Syndrome }\end{array}$ \\
\hline
\end{tabular}


2

3

4

5

6

7

8

9

10

11

12

13

14

15

16

17

18

19

20

21

22

23

24

25

28

29

30

31

32

33

34

35

36

37

38

39

40

41

42

43

44

45

46

47

48

49

50

51

52

53

54

55

56

57

58

59

60

URL: http:/mc.manuscriptcentral.com/gaut Email: pcasali@uthscsa.edu 


\title{
Autoimmune, rheumatic, chronic inflammatory diseases: Neutrophil Extracellular Traps on Parade
}

Malgorzata Justyna Podolska ${ }^{1 \#}$, Aparna Mahajan ${ }^{1 \#}$, Jasmin Knopf ${ }^{1 \#}$, Jonas Hahn ${ }^{1 \#}$, Sebastian Boeltz ${ }^{1}$, Luis Munoz ${ }^{1}$, Rostyslav Bilyy ${ }^{2^{*}} \&$ Martin Herrmann ${ }^{1}$

${ }^{1}$ Department of Internal Medicine 3- Rheumatology and Immunology, Friedrich-Alexander-Universität Erlangen-Nürnberg (FAU) and Universitätsklinikum Erlangen, Germany

${ }^{2}$ Danylo Halytsky Lviv National Medical University, Lviv, Ukraine

${ }^{\#} \mathrm{MJP}, \mathrm{AM}, \mathrm{JK}, \mathrm{JH}$ contributed equally to this work

"Correspondence:

Corresponding Author

Prof. Rostyslav Bilyy, r.bilyy@gmail.com

\begin{abstract}
Rheumatic diseases are a group of inflammatory conditions that affect joints and connective tissues and are often accompanied by pain and restriction of motility. In many of these diseases autoantibodies develop that react with molecules/structures commonly found hidden in neutrophils. Neutrophil extracellular trap (NET) formation and release is considered a defense mechanism against pathogens or endogenous danger signals and it has been associated with initial inflammatory responses. NETs are also endowed with an important resolution potential based on its intrinsic enzymatic activity, but in the case they are not timely removed from the crime scene they might modulate subsequent immune responses and contribute to the pathogenesis of chronic inflammatory diseases. In this review we will summarize the actual knowledge about the multifaceted roles of NETs in the etiology and pathogenesis of rheumatic autoimmune diseases.
\end{abstract}

\section{Rheumatic diseases}

Rheumatic diseases are a group of inflammatory conditions that affect joints and connective tissues and are often accompanied by pain and restriction of motility. A subgroup of these diseases prominates with multiorgan involvement and displays systemic appearance. Most of the rheumatic diseases have an important auto-inflammatory component with a dysregulation of several aspects of the immune system. One of these is autoimmunity which can be defined as the appearance of an active humoral and/or cellular immune response against own proteins (autoantigens) located in various tissues [1]. URL: http:/mc.manuscriptcentral.com/gaut Email: pcasali@uthscsa.edu 
These abnormalities together with long-term autoimmunity are thought to play decisive roles in the pathogenesis of rheumatic diseases. The most common rheumatic diseases with an strong autoimmune component include Rheumatoid Arthritis (RA), Psoriasis and Psoriatic Arthritis (PsoA), Systemic Lupus Erythematosus (SLE), Sjögren Syndrome, and Vasculitis [2]. Several diseasespecific features and potential pathophysiological mechanisms have been discovered for these diseases so far. The actual knowledge suggests that autoimmune diseases are multifactorial disorders showing high levels of complexity and heterogeneity although having a common pathogenesis: loss of self-tolerance $[3,4]$.

Intracellular autoantigens are usually not available for recognition by the immune system and are therefore by nature "foreign". Certain pathological conditions like an impaired clearance of dead and dying cells or an overflow of intracellular constituents into tissues might represent the driving force that triggers and/or fuels auto-inflammatory reactions and disease manifestations [5,6]. Neutrophil extracellular trap (NET) formation and release is considered a defense mechanism against pathogens [7]. This process has been associated with initial inflammatory responses and is also endowed with an important resolution potential based on its intrinsic enzymatic activity [8-10]. NETs are considered powerful enzymatic reactors and autoantigen repositories. In case they are not timely removed from the crime scene they might modulate subsequent immune responses.

In this review we will summarize the actual knowledge about the multifaceted roles of NETs in the etiology and pathogenesis of rheumatic autoimmune diseases.

Formation of Neutrophil Extracellular Traps (NET)

For decades neutrophilic granulocytes in the blood (referred to as neutrophils) have been known to patrol our tissues for pathogenic threats, phagocytose particulate matter, activate a cascade of reactive oxygen species (ROS) and destroy or neutralize pathogens. During the last 14 years neutrophils were demonstrated to utilize another mechanism to entrap dangerous bacteria and much bigger foreign objects ranging in size from nanoparticles to fungal hyphae [11]. The mechanism is executed by an active breakage of DNA-histone interactions, decondensation and release of DNA with the subsequent entrapment of particles by unbound chromatin decorated by the toxic proteinaceous content of neutrophils' granules [12]. Released decondensed DNA and histones have net-like appearance under the microscope and were termed neutrophil extracellular traps (NETs). Under certain conditions neutrophils reportedly survive NET release at least for a couple of hours [13]. 
We need to point that a suicidal type of death is quite reasonable for neutrophils, as these are terminally differentiated, cannot proliferate, and have a rather short half-life in the bloodstream (approximately $20 \mathrm{~h}$ ) [14]. Their heavily segmented nuclei allow them to easily exit capillaries and penetrate tissues with high velocity. Neutrophils patrol the epithelia of body surfaces and the lumina of various ducts [15]. They altruistically sacrifice themselves and release their toxic contents with the aim to stop bacterial invasions. NET formation is a reasonable protection mechanism and serves the aim to safeguard the body from aggressors. Released chromatin is not only able to directly immobilize and neutralize dangerous and numerically superior bacteria and stop their spreading, but also to enlace particles that are too big to be phagocytosed (fungal hyphae) [16]. NETs can sequester foreign and inert material like nanodiamonds [9], asbestos [17] and monosodium urate [18]. They can isolate toxic compounds e.g. from certain staphylococci and shield loci of necrosis and granulomas [19]. In some cases, NET-borne proteases are able to destroy proinflammatory cytokines and thus promote the resolution of inflammation $[18,20]$. Passivation of large/foreign/toxic objects is for sure beneficial to the organism, but it also challenges the immune system. Usually when NETs are released on relatively small areas they must be dismantled by enzymes like DNase-1 and DNase-1L3 $[21,22]$ and finally cleared by phagocytes, building a delicate balance between NET formation and their silent removal [23]. If clearance is disrupted either due to the overproduction of NETs or a failure of the phagocytic system, the organism will face a huge amount of chemically and enzymatically modified and thus immunologically "novel" epitopes. This is prone to result in the formation of antibodies to histones, DNA [5], cytoplasmic components of neutrophils (ANCA) [24], all being hallmarks for autoimmune disorders discussed below.

NETs in systemic lupus erythematosus (SLE)

Among many types of cell death the formation of neutrophil extracellular traps (NETs) is considered to be a novel source of autoantigens able to stimulate the chronic inflammatory responses in SLE $[25,26]$. The hallmark of this disease is the presence of autoantibodies against nuclear antigens, especially chromatin and dsDNA [27], main components of NETs. In 2010, Hakkim et al. reported that insufficient clearance of NETs leads to increased levels of anti-NET autoantibodies and this is translated into higher frequencies of lupus nephritis. It was found that serum DNase I was responsible for the dismantling of NETs in vivo. Sera of patients suffering from SLE showed decreased capacities of NET degradation. Two mechanisms were proposed to be involved in this process. On the one hand, the presence of DNase I inhibitors in sera of SLE patients is considered to be responsible for decreased clearance of harmful material. On the other hand, it was postulated that autoantibodies against NET components protect these structures from degradation [21]. It is consistent with previous observations demonstrating that number of SLE patients has DNase I inhibitory antibodies in their URL: http:/mc.manuscriptcentral.com/gaut Email: pcasali@uthscsa.edu 
sera [28] and that opsonization of DNA by anti-DNA antibodies protects from DNase digestion in vitro [29].

Additionally, in patients suffering from SLE a subset of so-called low density granulocytes (LDG), has been characterized. LDG are more potent in NET formation and induce cytotoxicity to endothelia through NET-associated proteases. Neutrophils forming NETs were preferentially detected in tissues of patients with SLE that had higher levels of circulating anti-dsDNA autoantibodies [30,31].

The inflammatory signature of patients with SLE is driven by type I interferon [32]. In 2011 it was shown that NETs are potent activators of IFN- $\alpha$ production by plasmacytoid dendritic cells (pDC), which in turn primes further neutrophils for additional release of NET [33,34]. This fuels a vicious cycle of inflammation and exacerbates the disease. NET-associated proteins can also efficiently activate NLRP3 inflammasome. In lupus macrophages this results in the release of II-1 $\beta$ and II-18, [35] which may cause the elevated II-18 levels reported in patients suffering from SLE [36,37]. The production of II-1 $\beta$ and II-18, in turn, induces formation of NETs creating a vicious positive feedback loop $[35,38]$.

Overall, these findings have provided evidence of the harmful role of NET forming neutrophils in the pathogenesis of inflammation of SLE.

NETs in rheumatoid arthritis (RA)

Anti-citrulline Antibodies (ACPAs) are the serological hallmark of rheumatoid arthritis (RA). Development of these autoantibodies is promoted by protein citrullination as a result of peptidyl arginin deiminases (PAD) activation. Importantly, the breaking of tolerance to citrullinated antigens already takes place before onset of the disease in genetically pre-disposed individuals and is triggered by environmental factors such as periodontitis [39-41] or in the lung of smokers [42-46]. These events give rise to a restricted ACPA repertoire that is further extended the clinical onset of disease by epitope spreading and affinity maturation [47,48]. In established RA, many antigens become targets of ACPAs only after deamination by PADs such as fibrinogen, vimentin, collagen II, filaggrin and histones [49]. Not only the synovium but also lungs [44,50,51] and myocardial tissue [52] contain many citrullinated proteins. Neutrophils and NETs contribute in two ways to this extended protein citrullination: (1) exposure of neutrophils to a variety of stimuli leads to deiminated histones [53,54] a major autoantibody target in RA [55-57], (2) granzyme B/perforin and complement activation including the formation of the membrane attack complex are able to induce extensive protein citrullination in synovial fluid neutrophils. The increased intracellular calcium in turn leads to increased PAD activity [58]. It has been shown that NET formation can be triggered by exposure to 
immunoglobulins of patients with RA or purified ACPA. Netting neutrophils can be detected in the synovial fluid and rheumatoid nodules of the patients [59]. Furthermore, increased levels of NET remnants were observed in the sera of patients with RA [60]. Mechanistically, it was shown in a mouse model of arthritis that excessive NET formation promotes Th1-mediated autoimmune responses via maturation of dendritic cell [61]. Therefore, the current hypothesis is that PAD activity due to extensive NET formation or bacterial origin (Porphyromonas gingivalis) leads to an excessive protein citrullination that serve as autoantigens in genetically pre-disposed individuals. However, which events finally lead to the break of tolerance against citrullinated proteins, how these events taking place in lung or oral cavity lead to destruction of joints and how this process is triggered in individuals without genetic predispositions are three cardinal questions that wait to be resolved.

NET formation in ANCA-associated vasculitides (AAV)

Anti-neutrophil cytoplasmic Autoantibody (ANCA) associated vasculitides (AAV) are systemic necrotizing small-vessel vasculitides, predominantly affecting intra-parenchymal small arteries, arterioles, capillaries, venules, and less often medium-sized arteries and veins [62]. They are classified into three distinct clinical diseases: granulomatosis with polyangiitis (GPA, formerly Wegener's granulomatosis), eosinophilic GPA (Churg-Strauss syndrome) and microscopic polyangiitis (MPA). However, they share immunopathologic hallmarks, e.g. the occurrence of ANCAs in $90 \%$ of patients with active disease. ANCAs are classified into cANCA and pANCAs according to their antigen specificity for proteinase 3 and myeloperoxidase, respectively [62]. Detection of these AAbs is used for the diagnosis of AAVs. Highly successful therapeutic usage of Anti-CD20 Antibodies promotes a role of ANCA and possibly further autoantibodies in the progression of AAVs [63].

A number of studies links the formation of NETs to disease onset, progression and perpetuation: (I) Neutrophils from AAV patients are more prone to release NETs spontaneously than neutrophils from healthy blood donors [64,65]; (II) NETs are released by ANCA-stimulated neutrophils; (III) Deposition of NETs in inflamed kidneys and circulating MPO-DNA complexes suggest that the interaction of NET with endothelia triggers vasculitis and precipitates the autoimmune response against neutrophil components in individuals with AAVs [66]; (IV) pANCAs and cANCAs target the NETs and the cell bodies of netting neutrophils, respectively [67]. (V) AAV patients have reduced DNasel activity resulting in impaired degradation of NETs [24]. The imbalance between NET formation and degradation is prone to increase the half-life of autoantigen presentation, which potentially enhances the exposure of the immune system to modified autoantigens and increases the capacity for NETinduced organ damage [68]. Intriguingly, ANCAs occur in $50 \%$ of patients with malaria. This supports the role of NETs in the etiopathogenesis of this, often fatal, disease. Malaria patients primarily 
develop pANCAs, supporting the notion that NETs may serve as an autoantigen also in this disease [69]. Whether NETs and pANCAs ultimately lead to (micro)-vascular occlusions deserve the attention of researchers.

\section{NETs in anti-phospholipid Syndrome (APS)}

Antiphospholipid syndrome is a systemic autoimmune disorder associated with elevated levels of antiphospholipid antibodies (aPL) [70] which precipitates as arterial and venous thrombosis and fetal loss. aPL, includes the Lupus anticoagulant (LA), IgG and/or IgM anticardiolipin (aCL) or IgG and/or IgM anti- $\beta 2$-glycoproteinl antibodies (anti-ß2-GPI) [71]. APS can be primary, in absence of other autoimmune diseases or secondary occurring with underlying autoimmune disease like SLE [70]. It has been known that aPL can promote thrombus formation by activating endothelial cells, monocytes and platelets. It is discussed that NETs contribute to thrombogenesis. Plasma samples from patients with APS reportedly harbor elevated levels of cell free DNA and NETs [72]. Yalavarthi et al. reported that neutrophils from patients with APS are more prone to spontaneous NET release and proposed a mechanism suggesting that $\mathrm{aPL}$ such as anti- $\beta 2-\mathrm{GPI}$ from patients with APS stimulate NET formation. They found a positive correlation between aPL and circulating levels of NETs. These increased levels of aPL results in more neutrophil activation and NET formation. aPL stimulated NET formation requires reactive oxygen species and is mediated via the stimulation of toll-like receptor 4 [72]. Murine model of APS suggested that, circulating activated neutrophils and NETs provide a scaffold for thrombus formation. Exaggerated thrombus formation was observed in mice treated with IgG from patients of APS compared to control. These thrombi displayed high amounts of citrullinated histone H3, a marker of canonical NETs [73]. Treatment with NETs dissolving DNase and neutrophil depletion antibodies reduced thrombus formation in murine models of APS [73]. Sera from patients with primary or secondary APS had decreased ability to degrade NETs as compared to healthy controls [74]. Patients with secondary compared to primary APS display a stronger negative correlation between degradation of NETs and antibodies against NET components as well as neutrophil remnants. However, decreased ability of NET degradation in APS has not yet been shown to be associated with levels of aPL. However, it has been associated with increased levels of antibodies against NETs and specific clinical manifestations like thromboses [74]. Increased NET formation mediated by autoantibodies in APS and decreased NET degradation in patients with APS suggests pathogenic roles of NETs in the thrombogenesis of patients with APS.

\section{NETs in Psoriasis}

Psoriasis is a chronic inflammatory systemic disease with a genetic basis, often characterized by patches of abnormal skin $[75,76]$. While clinical symptoms occur mostly on the skin, comorbidities such as URL: http:/mc.manuscriptcentral.com/gaut Email: pcasali@uthscsa.edu 
psoriatic arthritis, Crohn's disease, psychological/psychiatric disorders and uveitis often occur $[77,78]$. The exact etiology of psoriasis remains elusive, however important roles for Th1/Th17 cells have been attributed [76]. Neutrophils are among the first cells to enter the nascent psoriatic plaques [79] and have long been considered a hallmark of psoriasis. However, they have been primarily viewed only as effector cells. Recently, NETs have increasingly been implicated in the etiopathogenesis of psoriasis. In the early phase, LL37 binds to external DNA or RNA forming nucleic acid-LL37 complexes sensed by plasmacytoid $(\mathrm{pDC})$ and myeloid $(\mathrm{mDC})$ dendritic cells leading to downstream productions of type I interferons and TNF-a/IL-6, respectively. These pro-inflammatory cytokines foster the onset and clinical manifestations of psoriasis [80,81]. Further involvement of NETs was seen in a recent study which observed more NET forming neutrophils in the peripheral blood and skin lesions of patients with psoriasis showing a direct correlation with disease severity [82]. NETs reportedly induce the production of human b-defesin-2 (HBD-2) an antimicrobial peptide produced by epidermal keratinocytes which could explain the decreased susceptibility of psoriatic plaques to microbial infections [82]. Thus it is compelling to implicate NETs for providing the external DNA that drives inflammation in psoriasis. In addition to DNA-LL37 complexes a combination of neutrophil elastase and secretory leukocyte proteinase inhibitor (SLPI), both present in NETs, are also able to induce type 1 interferon via pDCs in psoriatic skin [83]. Thus it is no just a coincidence that the majority of IL-17 positive cells in psoriatic plaques are neutrophils and mast cells which themselves release IL-17 via external trap formation [84]. Although NET formation provides scaffolding for selfantigen presentation to the immune system it is not yet considered a defining feature in psoriasis. Instead NETs provide antimicrobial activities in psoriatic plaques and drive the onset and chronic inflammation in psoriasis.

Summarizing, NETs are involved in several autoimmune rheumatic diseases. They clog vessels and ducts; serve as autoimmunogen, and, especially in conditions of impaired clearance as autoantigen for the induction of interferon-driven anti-nuclear autoimmunity. Depending on the specific location and disease background NETs may turn out to be either beneficial or detrimental.

\section{Conflict of Interest}

All authors report no conflict of interest-financial or otherwise-that may directly or indirectly influence the content of the manuscript submitted.

\section{Funding}


The work was partially supported by the German Research Foundation (DFG, CRC1181-C03), by the EU H2020-MSCE-RISE-2015 project Nr. 690836 PANG, by the Volkswagen-Stiftung grant\# 90361, by the doctoral training program GK1660 of the DFG, grant from Ministry of Healthcare of Ukraine 0116U000759, and by Ardea Biosciences, Inc.

References:

1. Lleo, A., et al., Definition of human autoimmunity--autoantibodies versus autoimmune disease. Autoimmun Rev, 2010. 9(5): p. A259-66.

2. Hayter, S.M. and M.C. Cook, Updated assessment of the prevalence, spectrum and case definition of autoimmune disease. Autoimmun Rev, 2012. 11(10): p. 754-65.

3. Gaipl, U.S., et al., Clearance deficiency and systemic lupus erythematosus (SLE). J Autoimmun, 2007. 28(2-3): p. 114-21.

4. Munoz, L.E., M. Herrmann, and C. Berens, Dying autologous cells as instructors of the immune system. Clin Exp Immunol, 2015. 179(1): p. 1-4.

5. Mahajan, A., M. Herrmann, and L.E. Munoz, Clearance Deficiency and Cell Death Pathways: A Model for the Pathogenesis of SLE. Front Immunol, 2016. 7: p. 35.

6. Munoz, L.E., et al., The role of defective clearance of apoptotic cells in systemic autoimmunity. Nat Rev Rheumatol, 2010. 6(5): p. 280-9.

7. Brinkmann, V., et al., Neutrophil extracellular traps kill bacteria. Science, 2004. 303(5663): p. $1532-5$.

8. Biermann, M.H., et al., Oxidative Burst-Dependent NETosis Is Implicated in the Resolution of Necrosis-Associated Sterile Inflammation. Front Immunol, 2016. 7: p. 557.

9. Munoz, L.E., et al., Nanoparticles size-dependently initiate self-limiting NETosis-driven inflammation. Proc Natl Acad Sci U S A, 2016. 113(40): p. E5856-E5865. 
10. Munoz, L.E., et al., Missing in action-The meaning of cell death in tissue damage and inflammation. Immunol Rev, 2017. 280(1): p. 26-40.

11. Ermert, D., A. Zychlinsky, and C. Urban, Fungal and bacterial killing by neutrophils. Methods Mol Biol, 2009. 470: p. 293-312.

12. Manfredi, A.A., et al., The Neutrophil's Choice: Phagocytose vs Make Neutrophil Extracellular Traps. Front Immunol, 2018. 9: p. 288.

13. Yipp, B.G. and P. Kubes, NETosis: how vital is it? Blood, 2013. 122(16): p. 2784-94.

14. Silvestre-Roig, C., A. Hidalgo, and O. Soehnlein, Neutrophil heterogeneity: implications for homeostasis and pathogenesis. Blood, 2016. 127(18): p. 2173-81.

15. Leppkes, M., et al., Externalized decondensed neutrophil chromatin occludes pancreatic ducts and drives pancreatitis. Nat Commun, 2016. 7: p. 10973.

16. Zawrotniak, M., et al., Aspartic Proteases and Major Cell Wall Components in Candida albicans Trigger the Release of Neutrophil Extracellular Traps. Front Cell Infect Microbiol, 2017. 7: p. 414.

17. Desai, J., et al., Particles of different sizes and shapes induce neutrophil necroptosis followed by the release of neutrophil extracellular trap-like chromatin. Sci Rep, 2017. 7(1): p. 15003.

18. Schauer, C., et al., Aggregated neutrophil extracellular traps limit inflammation by degrading cytokines and chemokines. Nat Med, 2014. 20(5): p. 511-7.

19. Bilyy, R., et al., Neutrophil Extracellular Traps Form a Barrier between Necrotic and Viable Areas in Acute Abdominal Inflammation. Front Immunol, 2016. 7: p. 424.

20. Reinwald, C., et al., Erratum: Reply to "Neutrophils are not required for resolution of acute gouty arthritis in mice". Nat Med, 2017. 23(4): p. 526.

21. Hakkim, A., et al., Impairment of neutrophil extracellular trap degradation is associated with lupus nephritis. Proc Natl Acad Sci U S A, 2010. 107(21): p. 9813-8.

22. Jimenez-Alcazar, M., et al., Host DNases prevent vascular occlusion by neutrophil extracellular traps. Science, 2017. 358(6367): p. 1202-1206. 
23. Knight, J.S., C. Carmona-Rivera, and M.J. Kaplan, Proteins derived from neutrophil extracellular traps may serve as self-antigens and mediate organ damage in autoimmune diseases. Front Immunol, 2012. 3: p. 380.

24. Soderberg, D. and M. Segelmark, Neutrophil Extracellular Traps in ANCA-Associated Vasculitis. Front Immunol, 2016. 7: p. 256.

25. Bouts, Y.M., et al., Apoptosis and NET formation in the pathogenesis of SLE. Autoimmunity, 2012. 45(8): p. 597-601.

26. Pradhan, V.D., et al., Anti-neutrophil cytoplasmic antibodies (ANCA) in systemic lupus erythematosus: prevalence, clinical associations and correlation with other autoantibodies. J Assoc Physicians India, 2004. 52: p. 533-7.

27. Burlingame, R.W., et al., The central role of chromatin in autoimmune responses to histones and DNA in systemic lupus erythematosus. J Clin Invest, 1994. 94(1): p. 184-92.

28. Yeh, T.M., et al., Deoxyribonuclease-inhibitory antibodies in systemic lupus erythematosus. J Biomed Sci, 2003. 10(5): p. 544-51.

29. Emlen, W., R. Ansari, and G. Burdick, DNA-anti-DNA immune complexes. Antibody protection of a discrete DNA fragment from DNase digestion in vitro. J Clin Invest, 1984. 74(1): p. 185-90.

30. Villanueva, E., et al., Netting neutrophils induce endothelial damage, infiltrate tissues, and expose immunostimulatory molecules in systemic lupus erythematosus. J Immunol, 2011. 187(1): p. 538-52.

31. Denny, M.F., et al., A distinct subset of proinflammatory neutrophils isolated from patients with systemic lupus erythematosus induces vascular damage and synthesizes type I IFNs. J Immunol, 2010. 184(6): p. 3284-97.

32. Baechler, E.C., et al., Interferon-inducible gene expression signature in peripheral blood cells of patients with severe lupus. Proc Natl Acad Sci U S A, 2003. 100(5): p. 2610-5.

33. Lande, R., et al., Neutrophils activate plasmacytoid dendritic cells by releasing self-DNApeptide complexes in systemic lupus erythematosus. Sci Transl Med, 2011. 3(73): p. 73ra19.

34. Garcia-Romo, G.S., et al., Netting neutrophils are major inducers of type I IFN production in pediatric systemic lupus erythematosus. Sci Transl Med, 2011. 3(73): p. 73ra20. 
35. Kahlenberg, J.M., et al., Neutrophil Extracellular Trap-Associated Protein Activation of the NLRP3 Inflammasome Is Enhanced in Lupus Macrophages. Journal of Immunology, 2013. 190(3): p. 1217-1226.

36. Kahlenberg, J.M., et al., Inflammasome activation of IL-18 results in endothelial progenitor cell dysfunction in systemic lupus erythematosus. J Immunol, 2011. 187(11): p. 6143-56.

37. Calvani, N., et al., Up-regulation of IL-18 and predominance of a Th1 immune response is a hallmark of lupus nephritis. Clin Exp Immunol, 2004. 138(1): p. 171-8.

38. Mitroulis, I., et al., Neutrophil extracellular trap formation is associated with IL-1beta and autophagy-related signaling in gout. PLoS One, 2011. 6(12): p. e29318.

39. Chen, H.H., et al., Association between a history of periodontitis and the risk of rheumatoid arthritis: a nationwide, population-based, case-control study. Ann Rheum Dis, 2013. 72(7): p. 120611.

40. Maresz, K.J., et al., Porphyromonas gingivalis facilitates the development and progression of destructive arthritis through its unique bacterial peptidylarginine deiminase (PAD). PLoS Pathog, 2013. 9(9): p. e1003627.

41. Quirke, A.M., et al., Heightened immune response to autocitrullinated Porphyromonas gingivalis peptidylarginine deiminase: a potential mechanism for breaching immunologic tolerance in rheumatoid arthritis. Ann Rheum Dis, 2014. 73(1): p. 263-9.

42. Makrygiannakis, D., et al., Smoking increases peptidylarginine deiminase 2 enzyme expression in human lungs and increases citrullination in BAL cells. Ann Rheum Dis, 2008. 67(10): p. 1488-92.

43. Mclnnes, I.B. and G. Schett, The pathogenesis of rheumatoid arthritis. N Engl J Med, 2011. 365(23): p. 2205-19.

44. Lugli, E.B., et al., Expression of citrulline and homocitrulline residues in the lungs of nonsmokers and smokers: implications for autoimmunity in rheumatoid arthritis. Arthritis Res Ther, 2015. 17: p. 9.

45. Hosseinzadeh, A., et al., Nicotine induces neutrophil extracellular traps. J Leukoc Biol, 2016. 100(5): p. 1105-1112. 
46. Lee, J., et al., Nicotine drives neutrophil extracellular traps formation and accelerates collageninduced arthritis. Rheumatology (Oxford), 2017. 56(4): p. 644-653.

47. Sokolove, J., et al., Autoantibody epitope spreading in the pre-clinical phase predicts progression to rheumatoid arthritis. PLoS One, 2012. 7(5): p. e35296.

48. Johansson, L., et al., Antibodies directed against endogenous and exogenous citrullinated antigens pre-date the onset of rheumatoid arthritis. Arthritis Res Ther, 2016. 18(1): p. 127.

49. Corsiero, E., et al., NETosis as Source of Autoantigens in Rheumatoid Arthritis. Front Immunol, 2016. 7: p. 485.

50. Demoruelle, M.K., et al., Anti-Citrullinated Protein Antibodies Are Associated With Neutrophil Extracellular Traps in the Sputum in Relatives of Rheumatoid Arthritis Patients. Arthritis Rheumatol, 2017. 69(6): p. 1165-1175.

51. Demoruelle, M.K., et al., Antibody Responses to Citrullinated and Noncitrullinated Antigens in the Sputum of Subjects With Rheumatoid Arthritis and Subjects at Risk for Development of Rheumatoid Arthritis. Arthritis Rheumatol, 2018. 70(4): p. 516-527.

52. Giles, J.T., et al., Myocardial citrullination in rheumatoid arthritis: a correlative histopathologic study. Arthritis Res Ther, 2012. 14(1): p. R39.

53. Neeli, I., S.N. Khan, and M. Radic, Histone deimination as a response to inflammatory stimuli in neutrophils. J Immunol, 2008. 180(3): p. 1895-902.

54. Wang, Y., et al., Histone hypercitrullination mediates chromatin decondensation and neutrophil extracellular trap formation. J Cell Biol, 2009. 184(2): p. 205-13.

55. Pratesi, F., et al., Antibodies from patients with rheumatoid arthritis target citrullinated histone 4 contained in neutrophils extracellular traps. Annals of the rheumatic diseases, 2013: p. annrheumdis2012-202765.

56. Dwivedi, N., et al., Felty's syndrome autoantibodies bind to deiminated histones and neutrophil extracellular chromatin traps. Arthritis Rheum, 2012. 64(4): p. 982-92.

57. Corsiero, E., et al., Single cell cloning and recombinant monoclonal antibodies generation from RA synovial B cells reveal frequent targeting of citrullinated histones of NETs. Ann Rheum Dis, 2016. 75(10): p. 1866-75. 
58. Romero, V., et al., Immune-mediated pore-forming pathways induce cellular hypercitrullination and generate citrullinated autoantigens in rheumatoid arthritis. Sci Transl Med, 2013. 5(209): p. 209 ra150.

59. Khandpur, R., et al., NETs are a source of citrullinated autoantigens and stimulate inflammatory responses in rheumatoid arthritis. Sci Transl Med, 2013. 5(178): p. 178ra40.

60. Wang, W., W. Peng, and X. Ning, Increased levels of neutrophil extracellular trap remnants in the serum of patients with rheumatoid arthritis. Int J Rheum Dis, 2018. 21(2): p. 415-421.

61. Papadaki, G., et al., Neutrophil extracellular traps exacerbate Th1-mediated autoimmune responses in rheumatoid arthritis by promoting DC maturation. Eur J Immunol, 2016. 46(11): p. 25422554.

62. Jennette, J.C., et al., 2012 revised International Chapel Hill Consensus Conference Nomenclature of Vasculitides. Arthritis Rheum, 2013. 65(1): p. 1-11.

63. Lamprecht, P., et al., Pathogenetic and Clinical Aspects of Anti-Neutrophil Cytoplasmic Autoantibody-Associated Vasculitides. Front Immunol, 2018. 9: p. 680.

64. Soderberg, D., et al., Increased levels of neutrophil extracellular trap remnants in the circulation of patients with small vessel vasculitis, but an inverse correlation to anti-neutrophil cytoplasmic antibodies during remission. Rheumatology (Oxford), 2015. 54(11): p. 2085-94.

65. Grayson, P.C., et al., Neutrophil-Related Gene Expression and Low-Density Granulocytes Associated With Disease Activity and Response to Treatment in Antineutrophil Cytoplasmic AntibodyAssociated Vasculitis. Arthritis Rheumatol, 2015. 67(7): p. 1922-32.

66. Kessenbrock, K., et al., Netting neutrophils in autoimmune small-vessel vasculitis. Nat Med, 2009. 15(6): p. 623-5.

67. Panda, R., et al., Neutrophil Extracellular Traps Contain Selected Antigens of Anti-Neutrophil Cytoplasmic Antibodies. Front Immunol, 2017. 8: p. 439.

68. Gupta, S. and M.J. Kaplan, The role of neutrophils and NETosis in autoimmune and renal diseases. Nat Rev Nephrol, 2016. 12(7): p. 402-13.

69. Boeltz, S., et al., Neutrophil Extracellular Traps Open the Pandora's Box in Severe Malaria. Front Immunol, 2017. 8: p. 874. 
70. Bertolaccini, M.L., et al., 14th International Congress on Antiphospholipid Antibodies Task Force. Report on antiphospholipid syndrome laboratory diagnostics and trends. Autoimmun Rev, 2014. 13(9): p. 917-30.

71. Miyakis, S., et al., International consensus statement on an update of the classification criteria for definite antiphospholipid syndrome (APS). J Thromb Haemost, 2006. 4(2): p. 295-306.

72. Yalavarthi, S., et al., Release of neutrophil extracellular traps by neutrophils stimulated with antiphospholipid antibodies: a newly identified mechanism of thrombosis in the antiphospholipid syndrome. Arthritis Rheumatol, 2015. 67(11): p. 2990-3003.

73. Meng, H., et al., In Vivo Role of Neutrophil Extracellular Traps in Antiphospholipid AntibodyMediated Venous Thrombosis. Arthritis Rheumatol, 2017. 69(3): p. 655-667.

74. Leffler, J., et al., Degradation of neutrophil extracellular traps is decreased in patients with antiphospholipid syndrome. Clin Exp Rheumatol, 2014. 32(1): p. 66-70.

75. Nestle, F.O., D.H. Kaplan, and J. Barker, Psoriasis. N Engl J Med, 2009. 361(5): p. 496-509.

76. Girolomoni, G., U. Mrowietz, and C. Paul, Psoriasis: rationale for targeting interleukin-17. Br J Dermatol, 2012. 167(4): p. 717-24.

77. Pfohler, C., C.S. Muller, and T. Vogt, Psoriasis vulgaris and psoriasis pustulosa epidemiology, quality of life, comorbidities and treatment. Curr Rheumatol Rev, 2013. 9(1): p. 2-7.

78. Naldi, L. and S.R. Mercuri, Epidemiology of comorbidities in psoriasis. Dermatol Ther, 2010. 23(2): p. 114-8.

79. Christophers, E., G. Metzler, and M. Rocken, Bimodal immune activation in psoriasis. $\mathrm{Br} \mathrm{J}$ Dermatol, 2014. 170(1): p. 59-65.

80. Ganguly, D., et al., Self-RNA-antimicrobial peptide complexes activate human dendritic cells through TLR7 and TLR8. J Exp Med, 2009. 206(9): p. 1983-94.

81. Lande, R., et al., Plasmacytoid dendritic cells sense self-DNA coupled with antimicrobial peptide. Nature, 2007. 449(7162): p. 564-9.

82. Hu, S.C., et al., Neutrophil extracellular trap formation is increased in psoriasis and induces human beta-defensin-2 production in epidermal keratinocytes. Sci Rep, 2016. 6: p. 31119. 
83. Skrzeczynska-Moncznik, J., et al., Secretory leukocyte proteinase inhibitor-competent DNA deposits are potent stimulators of plasmacytoid dendritic cells: implication for psoriasis. J Immunol, 2012. 189(4): p. 1611-7.

84. Bley, T.A., et al., Noninvasive assessment of transstenotic pressure gradients in porcine renal artery stenoses by using vastly undersampled phase-contrast MR angiography. Radiology, 2011. 261(1): p. 266-73. 
Figure 1. Various kinds of particulate matter (like bacteria, fungi, viruses or nanoparticles) as well as certain chemicals (LPS, MSU, PMA, ionomycin, bicarbonate and $\mathrm{pH}$ ) can trigger NET formation. The released NETs then entrap pathogens, sequester necrotic foci and shield wounds; however in pathological conditions they may occlude vessels and ducts. If they are not cleared properly NETs may serve as nucleic acid containing autoantigen that fuel the interferon-driven chronic inflammation a hallmark for systemic lupus erythematosus, SLE. 
Figure 1. Various kinds of particulate matter (like bacteria, fungi, viruses or nanoparticles) as well as certain chemicals (LPS, MSU, PMA, ionomycin, bicarbonate and $\mathrm{pH}$ ) can trigger NET formation. The released NETs then entrap pathogens, sequester necrotic foci and shield wounds; however in pathological conditions they may occlude vessels and ducts. If they are not cleared properly NETs may serve as nucleic acid containing autoantigen that fuel the interferon-driven chronic inflammation a hallmark for systemic lupus erythematosus, SLE. 